\title{
Opposing impact of AID deficiency on BCL-2 and IL-6 driven B-lymphoma development in BALB/c mice
}

\author{
Chunyan $\mathrm{Gu}^{1 \Delta}$, Xuefang $\mathrm{Jing}^{1 \Delta}$, Ye Yang ${ }^{2^{*}}$, Siegfried Janz ${ }^{1 *}$ \\ ${ }^{I}$ Department of Pathology, Carver College of Medicine, The University of Iowa, Iowa City, Iowa, 52242, USA; \\ ${ }^{2}$ School of Medicine and Life Science, Nanjing University of Chinese Medicine, Nanjing, Jiangsu, 210023, China
}

\begin{abstract}
Activation-induced cytidine deaminase(AID), an essential enzymatic activity required for somatic hypermutation and immunoglobulin class switch recombination in the course of normal B-lymphocyte development, has been implicated in the initiation and promotion of malignant B-cell tumors by virtue of a complex mechanism that includes the generation of oncogene-activating genomic rearrangements and the introduction of point mutations in cancer genes. Here, we use transgenic mouse models of B-cell lymphoma driven by the pro-inflammatory cytokine, interleukin 6 (IL-6), or the survival-enhancing oncoprotein, B-cell leukemia 2 (BCL-2), to evaluate the impact of loss of AID on neoplastic B-cell development. We show that AID deficiency accelerates BCL-2 induced lymphoma but delays IL-6 induced lymphoma. This led us to conclude that AID may function as tumor suppressor or tumor promoter, depending on the genetic context. Elucidating the mechanism of AID's dual function during malignant B-cell transformation may be important for new approaches to tumor treatment and prevention.
\end{abstract}

Keywords: genetically engineered mouse model (GEMM) of human cancer, B-cell leukemia 2 (BCL-2), interleukin 6 (IL-6), activation-induced cytidine deaminase (AID)

\section{ABBREVIATIONS}

Aicda AID-encoding gene

AID activation-induced cytidine deaminase

BCL2 B cell leukemia 2 gene

BCL-2 B cell leukemia 2 oncoprotein

GEMM genetically engineered mouse model

Ig immunoglobulin

IgM immunoglobulin M

IL6 interleukin 6 gene

IL-6 interleukin 6

PCT plasma cell tumor or plasmactyoma

TME tumor microenvironment

*Correspondence to: Ye Yang, School of Medicine and Life Science, Nanjing University of Chinese Medicine, Nanjing, Jiangsu, 210023, China. TEL: +86-25-85811597, E-mail: yangye876@sina.com. Siegfried Janz, Department of Pathology, Carver College of Medicine, The

\section{INTRODUCTION}

Genetically engineered mouse models (GEMMs) of human neoplasms of the B-lymphocyte lineage, such as leukemia, lymphoma, plasmacytoma and myeloma, provide an important research tool for: (1) elucidating the biological pathways of neoplastic B-cell development, (2) discovering tumor-inhibiting suppressor or tumor-promoting oncogens, (3) interrogating the genetic and epigenetic makeup of tumor cells and last but not least, (4) evaluating the role of the tumor microenvironment (TME) in the natural history of malignant cell transformation ${ }^{[1]}$. In addition to addressing

University of Iowa, Iowa City, Iowa, 52242, USA. TEL:+1-319-3842869, E-mail: siegfried-janz@uiowa.edu.

${ }^{\Delta}$ C.G and X.J are co-first authors.

The authors reported no conflict of interest. 
fundamental knowledge gaps of this sort, GEMMs of human B-lineage malignancies contribute evaluable preclinical insights to the design and testing of new approaches for the treatment of patients with blood cancer. This may take the form of validating new molecular targets under the comparably straight forward, genetically defined and environmentally controlled conditions of a transgenic mouse study; assessing PK/ PD (pharmacokinetic and -dynamic) properties of candidate small-drug inhibitors and emerging immunotherapies; and evaluating new biomarkers for diagnostic and therapeutic purposes. The translational potential of B-lineage GEMMs is further underlined by the possibility to support ongoing clinical trials of promising new cancer therapies with dedicated preclinical co-trials using laboratory mice ${ }^{[2]}$. The broad utility of GEMMs provides a strong rationale for continuing with efforts both to develop models of human B-cell tumors for which none are available at this juncture, and to refine established models with regard to a more accurate representation of human disease features and an increased relevance for human cancer therapy and prevention.

We recently reported on the development of a GEMM of human Waldenström macroglobulinemia (WM) - a low-grade incurable IgM $^{+}$lymphoplasmacytic lymphoma (LPL) for which a laboratory animal model of de novo tumor development is lacking $^{[3]}$. Based on a large body of evidence that the proinflammatory cytokine, interleukin 6 (IL-6), and the survival-enhancing oncoprotein, B-cell leukemia 2 (BCL-2), play critical roles in the natural history of WM, we decided to evaluate whether the enforced expression of BCL-2 and IL-6 in mice unable to perform immunoglobulin class switch recombination may result in $\mathrm{IgM}^{+} \mathrm{B}$-cell tumors that mimic WM. To that end, we generated compound transgenic BALB/c (C) mice that harbored the human $B C L 2$ transgene, E $\mu \mathrm{SV}-$ BCL2-22 $2^{[4]}$, and the human IL6 transgene, H2- ${ }^{\mathrm{d}}-$ hIL $6^{[5,6]}$, on the genetic background of activationinduced cytidine deaminase (AID) deficiency ${ }^{[7]}$. We found that mice of this sort developed $\operatorname{IgM}^{+}$LPL-like tumors with full genetic penetrance ( $100 \%$ incidence) and relatively short onset (93 days median) ${ }^{[3]}$. In the course of these studies, we also made the surprising discovery that AID deficiency had an opposing impact on tumorigenesis: it accelerated BCL2-driven lymphoma but inhibited IL6-driven lymphoma.

Here we follow up on these findings with an updated study cohort, and present the time course of lymphoma development in AID-deficient BCL-2 transgenic mice $\left(\mathrm{BCL}^{+} \mathrm{AID}^{-}\right)$and $\mathrm{IL}-6$ transgenic mice (IL6 $6^{+} \mathrm{AID}^{-}$) — in both cases compared to their
AID-proficient counterparts: $\mathrm{BCL} 2^{+} \mathrm{AID}^{+}$mice and $\mathrm{IL}^{+} \mathrm{AID}^{+}$mice. We show that tumor development in $\mathrm{IL}^{+} \mathrm{AID}^{+}$mice is faster than in $\mathrm{IL}^{+} \mathrm{AID}^{-}$mice - $\mathrm{a}$ finding that is in sync with the widely held paradigm that AID is an oncogenic mutator protein that can initiate and promote lymphoma genesis by virtue of a complex mechanism that includes illegitimate genomic rearrangements and point mutations in immunoglobulin (Ig) and non-Ig genes ${ }^{[8-11]}$. We further demonstrate that lymphoma development in $\mathrm{BCL}^{+} \mathrm{AID}^{-}$ mice is accelerated relative to $\mathrm{BCL} 2^{+} \mathrm{AID}^{+}$controls, suggesting that AID functions as a tumor suppressor in this specific context. We describe gross and histopathological features of lymphoma-bearing, AIDdeficient $\mathrm{BCL}_{2}^{+}$and $\mathrm{IL}^{+}{ }^{+}$mice, including the surprising proclivity of strain $\mathrm{BCL} 2^{+} \mathrm{AID}^{-}$to concomitantly develop splenic histiocytic sarcoma. We also show that strain $\mathrm{IL}^{+} \mathrm{AID}^{-}$holds promise for modeling human $\operatorname{IgM}^{+}$plasma cell neoplasia and conclude with a brief discussion of the potential mechanism by which loss of AID function may promote BCL2-driven lymphoma yet inhibit IL6-driven lymphoma.

\section{MATERIAL AND METHODS}

\section{Genetic background and maintenance of mice}

All mice included in this study were on the genetic background of BALB/c (C). Strain BCL2 ${ }^{+}$IID $^{-}$(indicated by red arrow in Fig.1A) is homozygous for a null allele of the AID-encoding Aicda gene (AID $\left.{ }^{-}\right)^{[12]}$ and also carries a constitutively expressed human $B C L 2$ transgene, designated E $\mu \mathrm{SV}-\mathrm{BCL} 2-22$ (BCL2 $^{+}$;

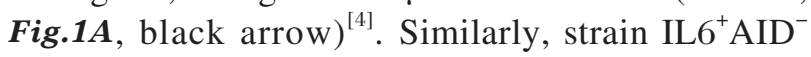
mice (Fig.1B, red arrow) harbor a constitutively expressed human IL6 transgene, designated H2-L ${ }^{\mathrm{d}}-\mathrm{IL} 6$ (IL6 $^{+} ;$Fig.1B, black arrow) ${ }^{[6]}$, on the genetic background of AID deficiency. Mice were housed in the University of Iowa (UI) Animal Resource Center under specific pathogen-free (SPF) conditions. This was important because previous studies using conventionally raised $A i c d a^{-l-} \mathrm{C}$ mice, resulted in unacceptably high death rates from a wasting syndrome that has been attributed to reactivation of latent viruses ${ }^{[9]}$ and an abnormal gut flora composition that leads to excessive lymphoid neogenesis ${ }^{[13]}$. A potential disadvantage of the SPF environment was the possible deceleration of inflammation- and IL-6-dependent lymphomagenesis, as exemplified by the striking reduction of peritoneal plasmacytoma(PCT) in SPF-raised C mice ${ }^{[14]}$. However, this study circumvented this disadvantage by relying on constitutively expressed and dominant transgenes: $\mathrm{BCL}^{+}$and $\mathrm{IL6}^{+}$. Mice were fed standard chow and received water ad libitum. All 
procedures involving mice, including tumor induction and euthanasia, observed strict ethical guidelines and were approved under IACUC Protocol 0701007.
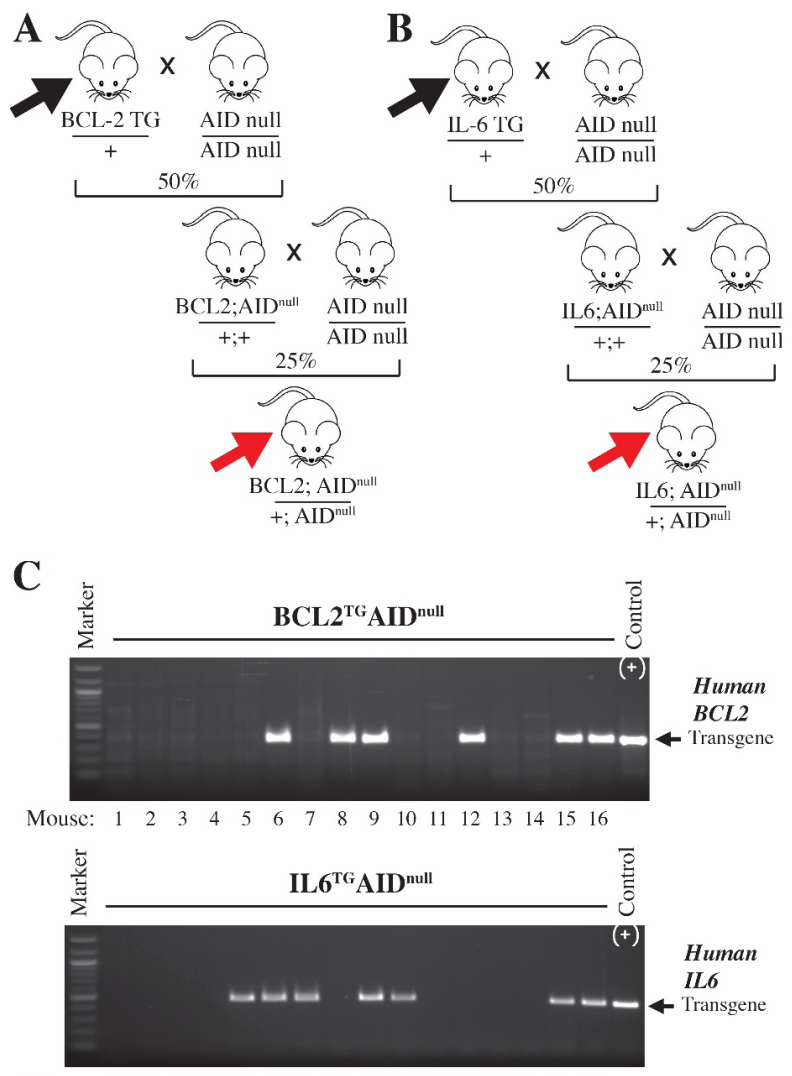

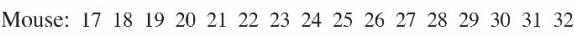

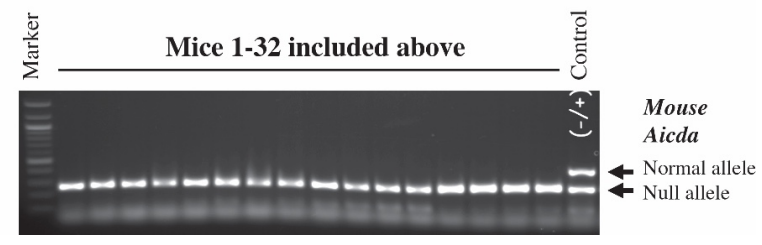

Fig. 1 Breeding and genotyping of $\mathrm{BCL}^{+} \mathrm{AID}^{-}$and IL6 $^{+} \mathbf{A I D}^{-}$mice.(A) Generation of $\mathrm{BCL}^{+} \mathrm{AID}^{-}$mice. Heterozygous transgenic $\mathrm{BCL} 2^{+}$mice were intercrossed with homozygous transgenic AID $^{\text {null }}$ mice. BCL2-transgenic offspring that carried one copy each of the AID $^{\text {null }}$ and wild-type allele were selected for a second round of breeding that involved a backcross on the genetic background of $\mathrm{AID}^{\text {null }}$. BCL2-transgenic pups that were homozygous for the AID ${ }^{\text {null }}$ allele were selected for studies on tumor development. (B) The same 2-step breeding strategy depicted above was used to make IL $6^{+}$AID $^{-}$ mice. Offspring of the desired genotypes were seen at the expected Mendelian frequency, just like in panel A. (C) Genotyping of mice relied on dedicated PCR assays for the BCL2 transgene (top panel) and IL6 transgene (center panel). A multiplex PCR assay using two different PCR primer pairs was employed to distinguish the AID-encoding Aicda wild-type gene from the non-coding Aicda null allele (bottom panel). All 32 mice included here were homozygous for the Aicda null allele and are, therefore, AID deficient (AID ${ }^{-}$). Mice 6, 8, 9, 12, 15 and 16 carrying the BCL2 transgene were included in the BCL2 ${ }^{+} \mathrm{AID}^{-}$study group. Mice 21-23, 25-26 and 31-32 harboring the IL6 transgene were included in the $\mathrm{IL}^{+} \mathrm{AID}^{-}$cohort.

\section{Generation of strain $\mathrm{BCL}^{+} \mathrm{AID}^{-}$and $\mathrm{IL}^{+} \mathrm{AID}^{-}$mice}

Both strains of mice were bred according to the schemes depicted in Fig.1A and 1B. The generation of $\mathrm{BCL}^{+} \mathrm{AID}^{-}$mice involved two steps. The first relied on intercrossing heterozygous transgenic $\mathrm{BCL} 2^{+}$mice and homozygous transgenic AID $^{\text {null }}$ mice (AID ${ }^{-}$) followed by selection of BCL2-transgenic offspring that carried one copy of the $A I D^{\text {null }}$ allele (inherited from the AID-deficient $A I D^{\text {null }}$ parent) and one copy of the Aicda wild-type allele (inherited from the AIDproficient BCL2-transgenic parent; designated by "+" symbol in the figure). $F_{1}$ offspring of this genotype were observed at the expected Mendelian frequency of $\sim 50 \%$. The second step relied on the backcross of $\mathrm{F}_{1}$ mice onto $\mathrm{AID}^{\text {null }}$, followed by selection of $B C L 2-$ transgenic pups that were homozygous for the AID $^{\text {null }}$ allele. Pups of this sort, designated BCL2 ${ }^{+} \mathrm{AID}^{-}$, occurred at a frequency of $\sim 25 \%$. The same 2 -step strategy was used to generate $\mathrm{IL}^{+} \mathrm{AID}^{-}$mice. These mice were also produced at the expected Mendelian frequency.

\section{Genotyping of mice}

PCR-based genotyping of mice took advantage of dedicated assays for the BCL2 and IL6 transgenes (top and center panel, respectively) and the AID-encoding Aicda wild-type and Aicda null alleles. PCR primer sequences, cycling conditions, and size of indicator fragments are provided in Table 1. Representative results of genotyping are shown in Fig.1C, which includes six BCL2 ${ }^{+} \mathrm{AID}^{-}$mice in the top panel (lanes 6 , $8,9,12,15$ and 16) and seven $\mathrm{IL}^{+} \mathrm{AID}^{-}$mice in the center panel (lanes 21-23, 25-26, 31-32). AID ${ }^{-}$status was demonstrated by detection of the Aicda null allele and lack of detection of the normal Aicda wild-type allele (bottom panel).

\section{Tumor diagnosis and histopathology}

Incipient lymphomas were detected by monitoring mice for splenomegaly and enlarged peripheral lymph nodes as well as declining health status parameters such as lethargy, hunched posture and poor grooming (rough hair coat). Mice were also evaluated for spontaneous diseases unrelated to lymphoma (e.g., rectal prolapse) and weight loss of more than $15 \%$ body weight and/or deviating $\geqslant 15 \%$ from agematched controls. At necropsy of mice, a standard panel of tissues, including lymphoid organs (lymph nodes, spleen) and parenchymatous organs (liver, kidney), was harvested, fixed in formalin and embedded in paraffin. Tissue sections $(4 \mu \mathrm{m})$ were deparaffinized, rehydrated and stained with hematoxylin and eosin 
Table 1 Primer sequences and cycling conditions for PCR-based genotyping of mice

\begin{tabular}{|c|c|c|c|c|c|}
\hline Gene & Forward primer & Reverse primer & $\begin{array}{c}\text { Cycling } \\
\text { conditions }^{1}\end{array}$ & $\begin{array}{c}\text { Cycle } \\
\text { number }^{2}\end{array}$ & $\begin{array}{l}\text { Indicator } \\
\text { fragment }\end{array}$ \\
\hline \multirow{3}{*}{$\begin{array}{c}B C L 2 \\
\text { transgene }\end{array}$} & \multirow{3}{*}{$\begin{array}{l}5^{\prime} \text { GCA GAC ACT CTA } \\
\text { TGC CTG TGT GG } 3^{\prime}\end{array}$} & $5^{\prime}$ GGA ACT GAT & $58^{\circ} \mathrm{C}, 30 \mathrm{~s}$ & \multirow{3}{*}{35} & \multirow{3}{*}{$350 \mathrm{bp}$} \\
\hline & & GAA TGG GAG CAG & $72^{\circ} \mathrm{C}, 45 \mathrm{~s}$ & & \\
\hline & & Т $3^{\prime}$ & $95^{\circ} \mathrm{C}, 30 \mathrm{~s}$ & & \\
\hline \multirow{3}{*}{$\begin{array}{c}I L 6 \\
\text { transgene }\end{array}$} & \multirow{3}{*}{$\begin{array}{l}5^{\prime} \text { ACC TCT TCA GAA } \\
\text { CGA ATT GAC AAA } 3\end{array}$} & $5^{\prime}$ AGC TGC GCA & $55^{\circ} \mathrm{C}, 30 \mathrm{~s}$ & \multirow{3}{*}{30} & \multirow{3}{*}{$451 \mathrm{bp}$} \\
\hline & & GAA TGA GAT GAG TTG & $72^{\circ} \mathrm{C}, 60 \mathrm{~s}$ & & \\
\hline & & Т $3^{\prime}$ & $95^{\circ} \mathrm{C}, 30 \mathrm{~s}$ & & \\
\hline \multirow{3}{*}{$\begin{array}{c}\text { Aicda } \\
\text { normal }\end{array}$} & $5^{\prime}$ CAA CGT GGC GTC & 5’ GGT CCC AGT CTG & $60^{\circ} \mathrm{C}, 30 \mathrm{~s}$ & \multirow{3}{*}{30} & \multirow{3}{*}{$400 \mathrm{bp}$} \\
\hline & CAA ACA GGC ACT & AGA TGT AGC GTA & $72^{\circ} \mathrm{C}, 30 \mathrm{~s}$ & & \\
\hline & TCC G 3 & GG $3^{\prime}$ & $95^{\circ} \mathrm{C}, 30 \mathrm{~s}$ & & \\
\hline \multirow{3}{*}{$\begin{array}{c}\text { Aicda } \\
\text { null }\end{array}$} & 5’AAC CAA GCC TAT & $5^{\prime} \mathrm{CTG}$ CCA AAC & $60^{\circ} \mathrm{C}, 30 \mathrm{~s}$ & \multirow{3}{*}{30} & \multirow{3}{*}{$260 \mathrm{bp}$} \\
\hline & GCC TAC AGC ATC & CTG ATG TCT TGA & $72^{\circ} \mathrm{C}, 30 \mathrm{~s}$ & & \\
\hline & CAG G $3^{\prime}$ & GTT TGA T $3^{\prime}$ & $95^{\circ} \mathrm{C}, 30 \mathrm{~s}$ & & \\
\hline
\end{tabular}

${ }^{1}$ One PCR amplification cycle consisted of primer annealing for $30 \mathrm{~s}$ at a relatively low temperature (line 1) followed by DNA polymerase-driven primer extension at $72^{\circ} \mathrm{C}$ (line 2) and melting of double-stranded DNA for $30 \mathrm{~s}$ at $95^{\circ} \mathrm{C}$ (line 3). Extension time varied, ranging from 30-60 s.

${ }^{2}$ PCR cycling was preceded by complete heat denaturation of template (genomic tail DNA) for 5 min at $95^{\circ} \mathrm{C}$ and ended with holding the reaction indefinitely at $4{ }^{\circ} \mathrm{C}$.

(H\&E). Additional sections were stained with Giemsa (according to the protocol of Lennert), PAS, or methylgreenpyronine, particularly in cases that required distinction between plasmacytosis (accumulation of normal-appearing and non-dividing plasma cells), plasma cell hyperplasia (mixtures of normal and aberrant, hyperchromatic, sometimes mitotically active plasma cells, micro-plasmacytoma (isolated clusters of malignant plasma cells, presumptive precursors of overt PCT), frank PCT, and anaplastic PCT (mixture of immunoblasts and plasma cells with $>90 \%$ of cells being immature forms of malignant plasma cells).

\section{RESULTS}

\section{AID deficiency accelerates BCL2-driven B- cell neoplasms}

To determine whether enforced transgenic expression of BCL2 acts synergistically with loss of AID to promote the malignant transformation of $\mathrm{B}-$ lymphocytes, AID-deficient BALB/c (C) BCL2 ${ }^{+} \mathrm{AID}^{-}$ mice were monitored at weekly intervals for signs of tumor development. This included declining health status parameters and palpable splenomegaly and peripheral lymph node enlargement. Twenty-one of $21(100 \%)$ mice developed tumors and the median survival of mice was 216 days. The range of survival was 115-312 days (Fig.2A, red curve). Mean tumor onset and standard deviation of the mean (SD) were (206.0 \pm 53.5$)$ days (Fig.2C, left). Tumor development in the AID-proficient control group of BCL2 ${ }^{+} \mathrm{AID}^{+}$ mice $(n=14)$ was significantly slower (384 days median survival; range 327-623 days) based on MantelCox log-rank analysis $(P<0.0001 ;$ Fig.2A, black curve). Slow tumor development was also reflected in late mean tumor onset in $\mathrm{BCL}^{+} \mathrm{AID}^{+}$mice, $(421.0$ $\pm 91.3)$ days, which was significantly different from that in their $\mathrm{AID}^{-}$counterparts $\left(P<10^{-3}\right.$, Student's $t$ test; Fig.2C, left). Accelerated tumor onset in the $\mathrm{AID}^{-}$cohort was surprising given that -in agreement with the well-established mutagenic function of activation-induced cytidine deaminase ${ }^{[15]}$ - AID proficiency has been demonstrated to promote mouse Blineage cancers in other studies ${ }^{[9,10]}$.

\section{AID deficiency decelerates IL6-driven B-cell neoplasms}

In parallel to the study described above, tumor development was determined in two cohorts of AID deficient or proficient IL $6^{+}$mice. Mice were evaluated for tumor manifestation using the same metrics as in the BCL2 study. IL6 ${ }^{+}$AID $^{-}$mice $(n=20)$ developed tumors with full genetic penetrance (100\% incidence). Median survival was 325 days (range141-351 days; Fig.2B, red curve). Mean tumor onset was (305.0 \pm 50.7) days (Fig.2C, right). Tumor development in the AID-proficient control group $\left(\mathrm{IL}^{+} \mathrm{AID}^{+} ; n=22\right)$ was significantly faster than in $\mathrm{AID}^{-}$mice (220 days median survival; range 130-465 days), using logrank analysis for statistical comparison $(P=0.0003$; Fig.2B, black curve). In accordance with that, mean tumor onset in IL $6^{+} \mathrm{AID}^{+}$mice was significantly faster $[(238.0 \pm 76.5)$ days $]$ than in the IL6 $^{+}$AID $^{-}$cohort $(P<$ $10^{-3}$, Student's $t$ test; Fig.2C, right). Delayed tumor onset in $\mathrm{AID}^{-}$mice was consistent with the mutagen$\mathrm{ic}^{[15]}$ and tumor-promoting role of AID in the B-cell lineage ${ }^{[9,10]}$ mentioned above. 
A

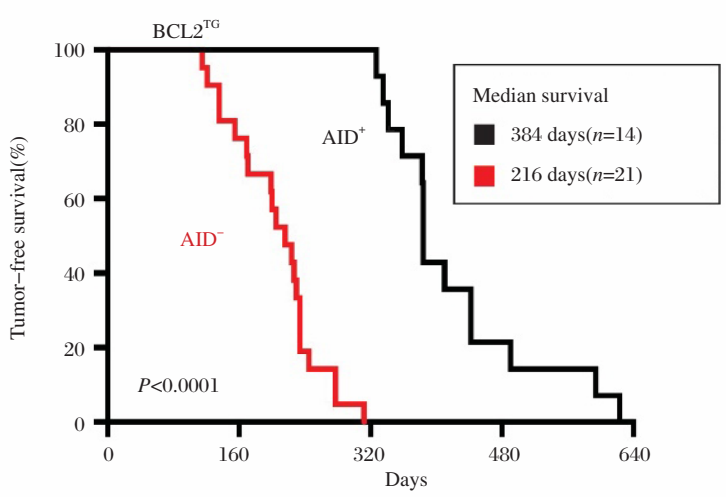

B

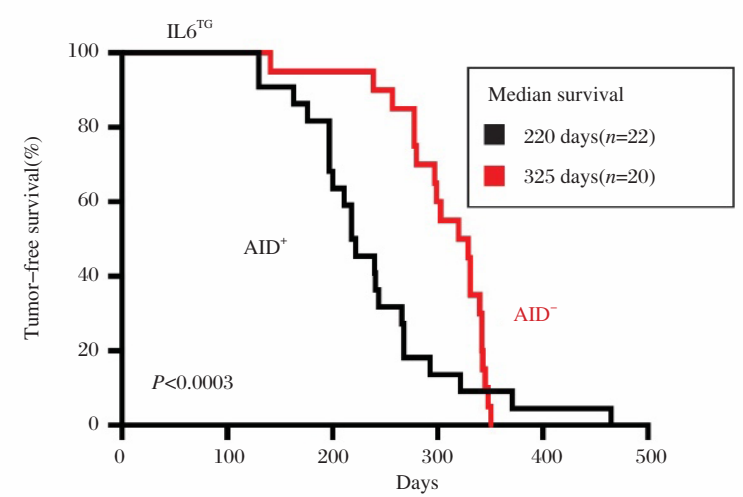

C

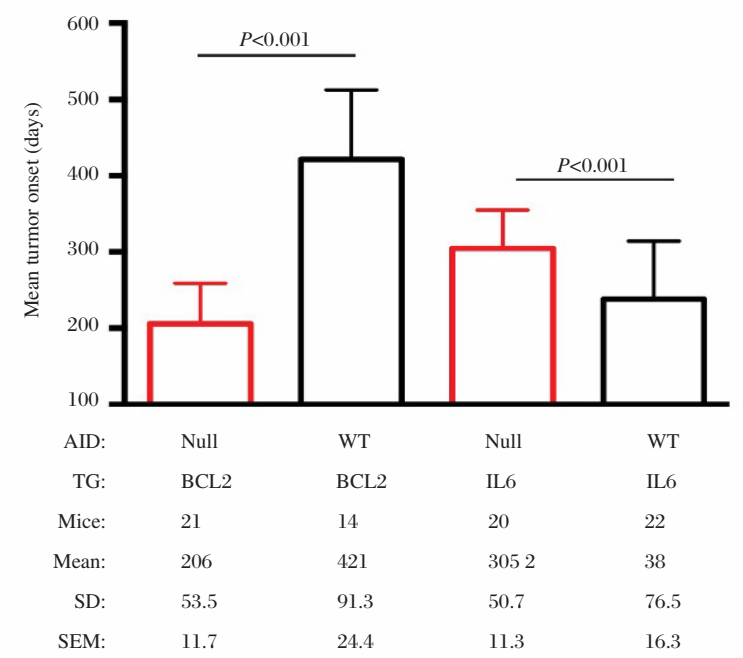

Fig. 2 Tumor-free survival of AID-proficient $\left(\mathbf{A I D}^{+}\right)$or AID-deficient $\left(\mathrm{AID}^{-}\right) \mathrm{BCL2}^{+}$and $\mathrm{IL6}^{+}$mice on the genetic background of BALB/c (C). (A) Kaplan-Meier survival curve of $\mathrm{BCL}^{+} \mathrm{AID}^{+}$and $\mathrm{BCL}^{+} \mathrm{AID}^{-}$mice. AID status, number of mice, and median survival of mice are indicated. Log rank analysis demonstrated a strikingly reduced survival of AIDdeficient mice compared to AID-proficient counterparts $(\mathrm{P}<$ $0.0001)$. (B) Survival of $\mathrm{IL}^{+} \mathrm{AID}^{+}$and $\mathrm{IL}^{+} \mathrm{AID}^{-}$mice. AIDdeficient mice lived significantly longer than AID-proficient controls $(\mathrm{P}=0.0003)$. (C) Mean tumor onset of the 4 groups of mice included in panels A and B. Standard deviation (SD) and standard error of the mean (SEM) are also indicated. Statistical comparison using Student's t test revealed that AID deficiency promotes BCL2 $2^{+}$tumors but inhibits IL6 ${ }^{+}$tumors.
$\mathrm{BCL}^{+} \mathrm{AID}^{-}$and $\mathrm{IL6}^{+} \mathrm{AID}^{-}$mice are prone to lymphoma

The tumor diagnosis of $\mathrm{BCL}^{+} \mathrm{AID}^{-}$and $\mathrm{IL}^{+} \mathrm{AID}^{-}$ mice was confirmed in all cases by gross pathological findings at necropsy, invariably including pronounced splenomegaly and generalized lymph node enlargement (Fig.3A). The mean weight of the spleen was more than 10 fold increased in both strains of mice: in BCL2 transgenics [(1.44 \pm 0.88$) \mathrm{g} ; n=17$ ] a bit more than in IL6 transgenics [(1.15 \pm 0.77$) \mathrm{g}$; $n=15]$, but this was not significant by Mann-Whitney $t$ testing(Fig.3B). A peculiar feature of tumorbearing $\mathrm{IL}^{+} \mathrm{AID}^{-}$mice, which had not been seen in the $\mathrm{BCL} 2^{+} \mathrm{AID}^{-}$cohort, was severe and widespread abnormalities of the venous circulatory system. This included stark dilation of, and apparent stasis in large veins that was particularly obvious in subcutaneous and major abdominal veins including mesenteric blood vessels (Fig.3C, first two panels on the left). Thickness of blood, venous thrombosis, and thromboembolism may in fact be the underlying reason for extensive coagulative necrotic changes of body tips, such as ears and toes, frequently seen in IL6 ${ }^{+} \mathrm{AID}^{-}$mice $($Fig.3C, center). Nodular enlargements of lymphoid follicles in the gut wall, generating a phenotype resembling "pearls on a chain" were also repeatedly observed (Fig.3C, right).

\section{Histopathological features common to $\mathrm{BCL2}^{+} \mathrm{AID}^{-}$and $\mathrm{IL6}^{+} \mathrm{AID}^{-}$mice}

Histological examination of $\mathrm{H} \& \mathrm{E}-$ stained tissue sections of tumor-bearing $\mathrm{BCL}^{+} \mathrm{AID}^{-}$and $\mathrm{IL}^{+} \mathrm{AID}^{-}$ mice demonstrated widespread systemic disease involving both lymphoid and non-lymphoid tissues (Fig.4). At diagnosis, all mice had progressed to an advanced stage at which Peyer's patches were visibly swollen with normal lymph node structures completely effaced by homogeneous sheets of tumor cells. This is illustrated in the lower part of the figure, using a $\mathrm{BCL}^{+} \mathrm{AID}^{-}$mouse as example. The upper part of the figure depicts disseminated lymphoma cell infiltrates in the liver and lung, taking an IL6 ${ }^{+}$AID $^{-}$mouse as example. Similar changes were seen in bone marrow and other tissue sites (not shown). Tumor cells were $\mathrm{B} 220^{+}$by immunohistochemistry (not shown) and therefore of $\mathrm{B}$ cell origin, as described in greater depth the Bethesda proposal for classifying lymphoid neoplasms in laboratory mice ${ }^{[16]}$. Severe kidney disease was also common to both strains of mice (Fig.5). Changes included cortical focal infiltrates of lymphoma cells that resulted in glomerular damage that included significant loss of cells, expansion of the me- 
A

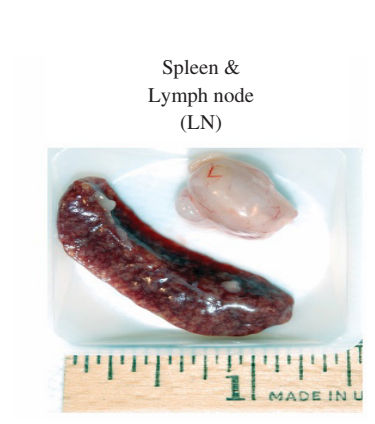

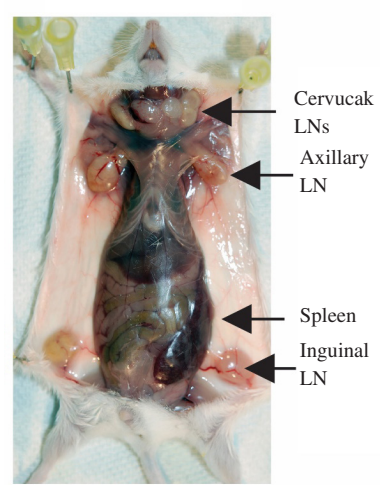

B

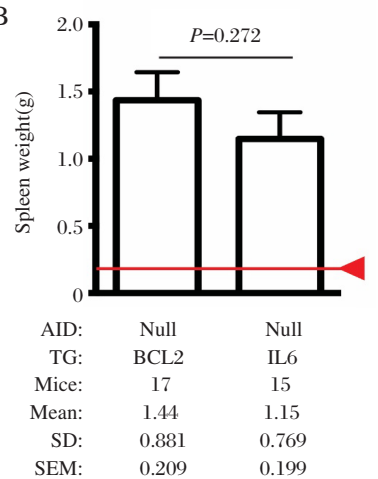

$\mathrm{C}$

Subcutenous vein

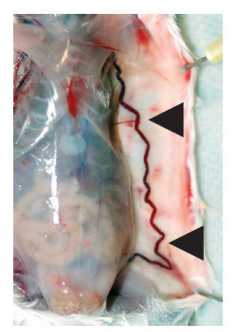

Gut loop with mesentery

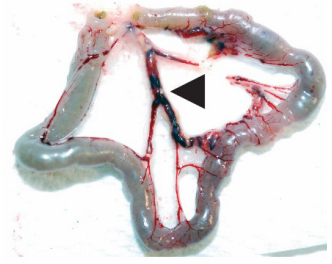

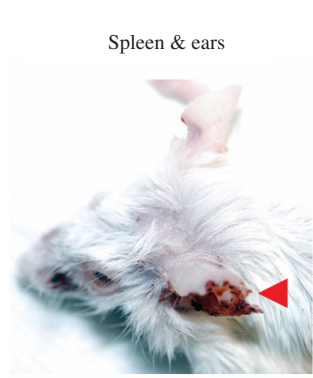

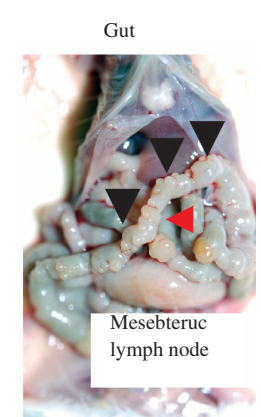

Fig.3 Gross pathology of tumor-bearing BCL2 $^{+} \mathbf{A I D}^{-}$and IL6 $^{+} \mathbf{A I D}^{-}$mice. (A) Necropsy photograph of a representative BCL2 ${ }^{+} \mathrm{AID}^{-}$ mouse with advanced disease. Enlarged cervical, axillary and inguinal lymph nodes are indicated by black arrows pointing left. The massively enlarged, $~ 1.5$ inch long spleen, which shines through the peritoneal wall and is also indicated by arrow, is shown next to a ruler on the left. Lymphomaharboring $\mathrm{IL}^{+} \mathrm{AID}^{-}$mice presented with the same features (not shown). (B) Mean spleen weights in the BCL2 $2^{+} \mathrm{AID}^{-}$cohort [(1.44 \pm 0.86$) \mathrm{g}$; range $0.4-2.9 \mathrm{~g} ; n=17]$ and $\mathrm{IL}^{+} \mathrm{AID}^{-}$cohort $[(1.15 \pm 0.77) \mathrm{g}$; range $0.4-2.5 \mathrm{~g} ; n=14]$ were much higher than seen in normal $\mathrm{C}$ mice $(\sim 0.1 \mathrm{~g}$, indicated by horizontal red line and red arrow head), but were not different by Student's $t$ test $(P=0.272)$. (C) Peculiar features of tumor-bearing IL6 ${ }^{+} \mathrm{AID}^{-}$mice. These mice repeatedly presented with changes that were not seen in any other of the strains included in panel a. Changes included apparent circulatory and/or blood coagulation problems that resulted in dilated blood vessels in the subcutis (indicated by arrowheads in leftmost image), the mesentery $\left(2^{\text {nd }}\right.$ image; mesenteric blood vessel containing thrombi, arrowhead) and many other tissue sites (not shown). Similar problems in the microcirculation may be the underlying reason for necrotic body tips including ears ( $3^{\text {rd }}$ image; necrotic, frizzled and hemorrhagic ears) and toes (not shown). Another peculiarity of strain $\mathrm{IL}^{+} \mathrm{AID}^{-}$was pronounced nodular lymphoid growth in the gut wall, leading to changes reminiscent of "pearls on a chain", shown in the rightmost image.

sangial matrix and marked interstitial fibrosis. Similar changes are typically found in severe kidney disease in patients with myeloma or related blood disorders prone to producing large amounts of free immunoglobulin light chains (IgL) and excreting them renally. IgL cast nephropathy was more pronounced in strain $\mathrm{IL}^{+} \mathrm{AID}^{-}$than $\mathrm{BCL2}^{+} \mathrm{AID}^{-}$mice. Kidney tubules were dilated and largely clogged up with eosinophilic proteinaceous casts (yellow arrow in the $10 \times$ image) that were also detected in glomeruli (yellow arrows in the $100 \times$ image).

\section{Histopathological findings unique to BCL2 $^{+} \mathrm{AID}^{-}$or $\mathrm{IL6}^{+} \mathrm{AID}^{-}$mice}

A somewhat unexpected but recurrent observation in $\mathrm{BCL}^{+} \mathrm{AID}^{-}$mice was the co-existence of lymphoma and histiocytic sarcoma (HS, Fig.6). HS was found in the spleen and/or lymph nodes, usually more advanced in the former tissue site in cases in which it occurred in both. HS was readily recognized at low- magnification microscopy due to its hallmark multinucleated giant cells that often contained more than 20 nuclei in a horseshoe-like arrangement. Considering strong molecular evidence for a common clonal origin of B-lymphoma and HS in humans; e.g., in patients with follicular lymphoma ${ }^{[17]}$, it is possible that HS in strain $\mathrm{BCL}^{+} \mathrm{AID}^{-}$is also derived from a common progenitor. However, this has not been demonstrated. A striking feature of $\mathrm{IL}^{+} \mathrm{AID}^{-}$mice, not seen in strain $\mathrm{BCL}^{+} \mathrm{AID}^{-}$, was the large number of aberrant plasmablasts and plasma cells in multiple tissue sites. This is depicted in Fig.7 using the spleen of a tumorbearing IL $^{+} \mathrm{AID}^{-}$mouse as an example. Extensive sheets of abnormal plasma cells populate peri- and interfollicular areas in the white pulp, suggesting a diagnosis of plasmacytoma at higher magnification. These cells frequently contained Russell bodies; i.e., large cytoplasmic globules of antibodies (immunoglobulin) that, for reasons that are unclear, cannot be secreted into the extracellular milieu. A related finding 


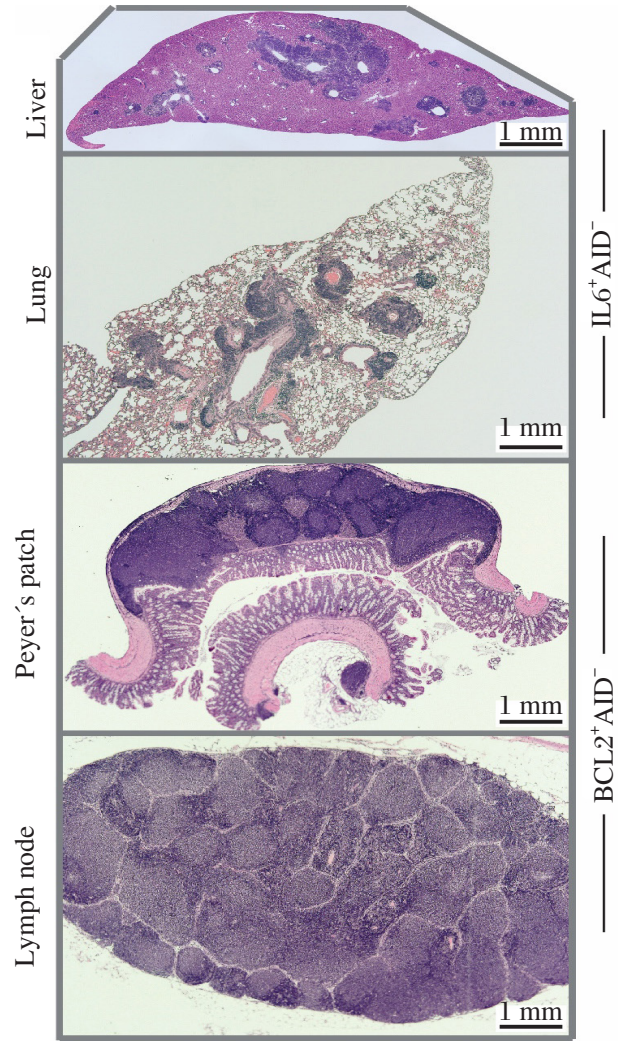

Fig.4 Histopathology of malignant lymphoma. Shown are lowpower photomicrographs of hematoxylin and eosin (H\&E) stained FFPE (formalin-fixed paraffin embedded) tissue sections from 2 tumor-bearing $\mathrm{IL6}^{+} \mathrm{AID}^{-}$mice (upper part) and 2 tumor-bearing $\mathrm{BCL}^{+} \mathrm{AID}^{-}$mice (lower part) to illustrate the tumor dissemination pattern in lymphoid and non-lymphoid tissues. Note the predominantly periportal and peribronchial/perivascular infiltration of tumor cells in liver and lung, respectively. The Peyer's patch, which is only moderately enlarged in this case, exhibits abnormal, enlarged lymphoid follicles at different stages of tumor progression. The normal lymph node architecture of the node shown at bottom is replaced by the pseudo-follicular, nodular expansion of malignant B cells and plasma cells. in $\mathrm{BCL}^{+} \mathrm{AID}^{-}$mice (not shown) was Mott cells-a typical plasma cells that harbor multiple immunoglobulin inclusion bodies that look like grape vines. Strain IL6 $^{+}$AID $^{-}$may be a good GEMM of human IgM $^{+}$ plasmacytoma/myeloma, rare diseases for which accurate experimental model systems do not exist. Additional work is warranted to evaluate this possibility, which should include a detailed comparison of tumor development and tumor phenotypes in $\mathrm{IL}^{+} \mathrm{AID}^{-}$and $\mathrm{IL6}^{+} \mathrm{AID}^{+}$mice (Fig.8). This shall be the reported in a follow-up publication at a later time.

\section{DISCUSSION}

This study took advantage of the E $\mu \mathrm{SV}-\mathrm{Bcl}-2-22$ $\left(\mathrm{BCL}^{+}\right)^{[4]}$ and H2-Ld-IL6 $\left(\mathrm{IL6}^{+}\right)^{[6,21]}$ transgenes to evaluate the role of AID in lymphoma development. We performed the study on the genetic background of BALB/c (C) because a large body of tumor induction results ${ }^{[22]}$ taught us that, due to a complex genetic trait ${ }^{[23-25]}$, strain $\mathrm{C}$ is highly susceptible to latestage $\mathrm{B}$ cell and plasma cell neoplasms ${ }^{[26]}$, including those promoted by BCL-2 ${ }^{[27]}$ and IL- $6^{[28]}$. We relied on $\mathrm{BCL}^{+}$for tumor promotion because it is well established that BCL-2 - the founding member of an evolutionarily conserved family of proteins that share BH (BCL-2 homology) domains and either promote or inhibit apoptosis (programmed cell death) by means of regulating mitochondrial outer membrane permeabilization (MOMP) - is a death repressor that is crucial for the malignant transformation of $\mathrm{B}$ cells and plasma cells. Enforced expression of BCL-2 promotes lymphoma in mice by virtue of increasing apoptotic thresholds in tumor precursors ${ }^{[29]}$. This is in line with

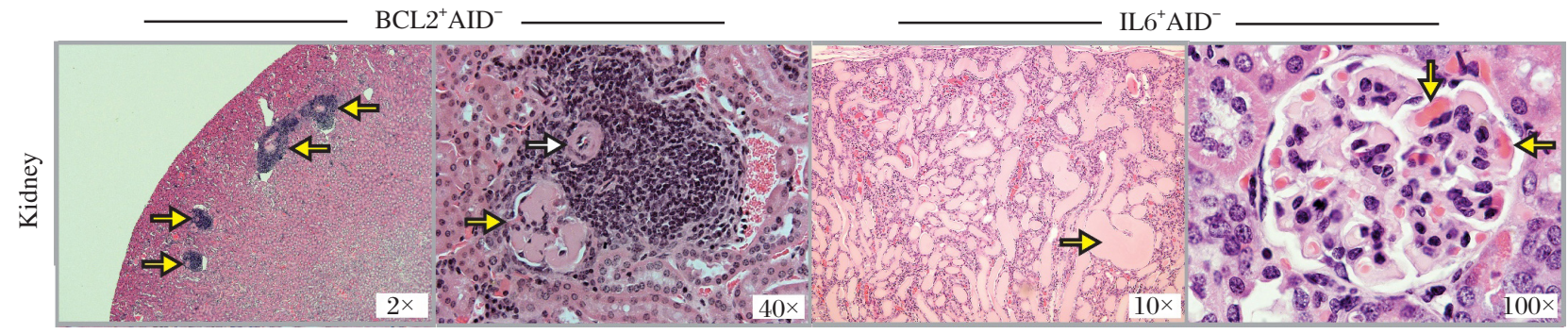

Fig.5 Histopathology of kidney disease. Presented in the upper half of the image is an example of the type of tumor cell infiltration in the cortex of the kidney (indicated by yellow arrows) seen in strain BCL2 AID $^{-}$mice. Four dense periglomerular foci of lymphoma cells are present in the overview image $(2 \times$ original magnification). The higher-magnification image $(40 \times)$ reveals a damaged glomerulus exhibiting a significant loss of cells, prominent interstitial fibrosis and a distinctive nodular appearance caused by expansion of the mesangial matrix. Similar changes are typically found in humans with myeloma kidney disease due to the deposition of PAS (periodic acid-Schiff) positive paraprotein. Shown in the lower half of the image panel is a low $(10 \times)$ and high $(100 \times)$ magnification view of the apparent light-chain cast nephropathy that was often found in strain IL6 $^{+}$AID $^{-}$mice. The tubules are dilated and essentially clogged up with eosinophilic proteinaceous casts, that have a crystalline appearance (yellow arrow in the $10 \times$ image). Casts of this sort are also present in glomeruli (yellow arrows in the $100 \times$ image). Similar changes are seen in patients with systemic Ig light-chain deposition disease. 

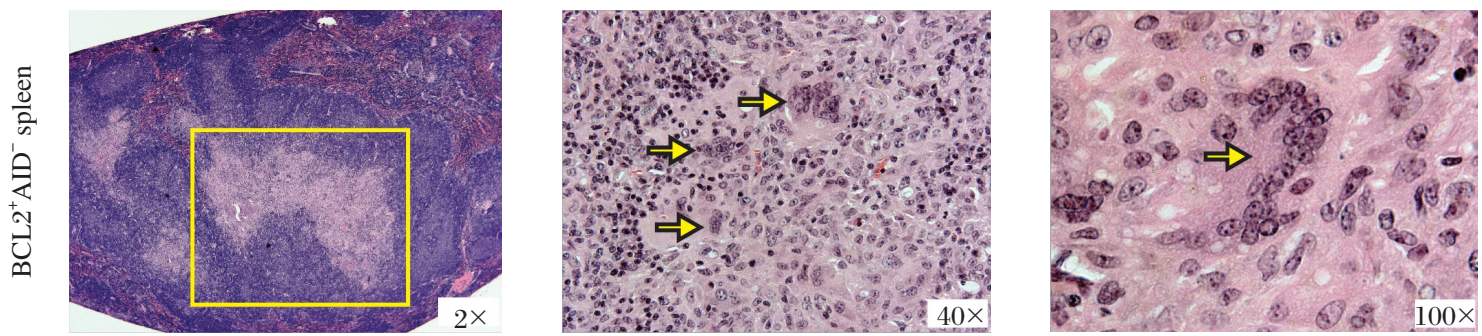

Fig.6 Histiocytic sarcoma (HS) in BCL2 ${ }^{+}$AID $^{-}$mice. The splenic HS found in this mouse corresponds to the pale region in the $2 \times$ image indicated by the yellow rectangle. The center image $(40 \times)$ presents a portion of the sarcoma that contains 3 multinucleated tumor giant cells, a hallmark of HS. The cells are labeled with yellow arrows pointing right. Another multinucleated giant cell with more than 25 nuclei (arrow) is shown at high power at the bottom $(100 \times)$.
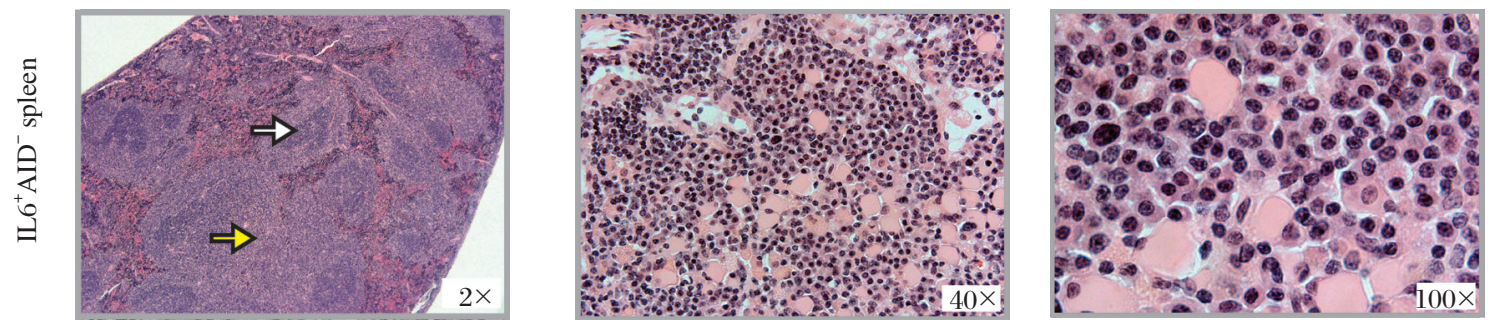

Fig.7 Atypical plasma cells in IL6 $^{+} \mathbf{A I D}^{-}$mice. Extensive peri- and interfollicular plasma cell accumulations (yellow arrow) in the white pulp of a spleen of an IL6-transgenic mouse $(2 \times)$. A somewhat darker B-cell follicle that consists of tightly packed, small lymphocytes is indicated by white arrow for comparison. Many plasma cells in the $40 \times$ image contain Russell bodies - cytoplasmic, eosinophilic, homogeneous globules (inclusion bodies) that consist in large measure of immunoglobulin that cannot be secreted to the extracellular milieu. Mott cells; i.e., atypical plasma cells containing multiple but relatively small Russell body—like globules, were also frequently encountered (not shown).
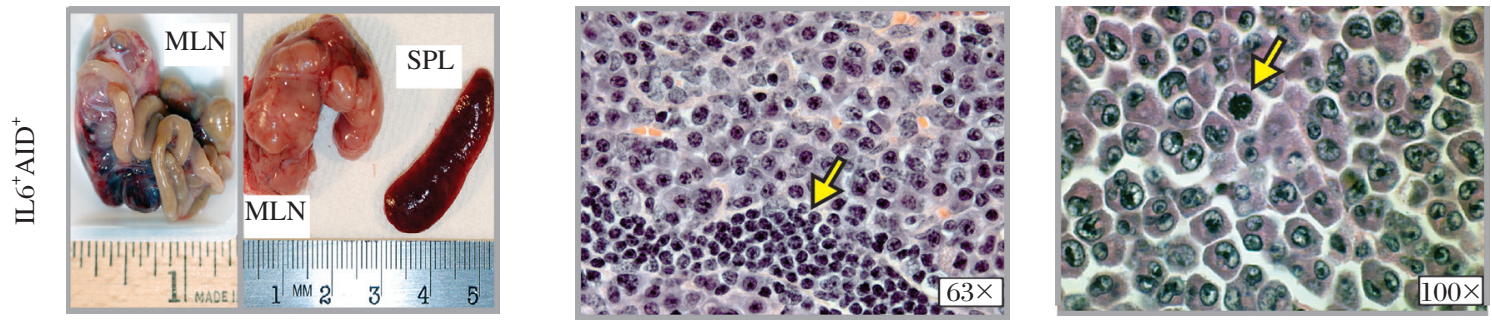

Fig.8 Frank plasma cell neoplasia in $\mathbf{I L 6}^{+} \mathbf{A I D}^{+}$mice. Shown in the left are autopsy findings in 2 tumor-bearing mice that harbored massively enlarged mesenteric lymph nodes (MLN) loaded with neoplastic plasma cells. The spleen is included in one case to depict the pronounced splenomegaly that is consistently seen in these cases. The rulers in the left and right hand images use the imperial inch and international centimeter scale, respectively. The center panel presents a photographic image of an H\&E-stained tissue section of a cervical lymph node heavily infiltrated with malignant plasma cells. A streak of normal lymphocytes, indicated by yellow arrow, is included for size comparison ( $63 \times$ original magnification). Depicted in the right panel is the "wall-to-wall" infiltration of an inguinal lymph node with a malignant plasma cell tumor. The yellow arrow pointing down denotes a tumor cell undergoing mitosis. Normal lymph node architecture was completely effaced (H\&E, 100×).

evidence that BCL-2 blocks many death signals in normal $\mathrm{B}$ cells ${ }^{[30,31]}$, cooperates with oncogenes in vitro to promote mitogen-independent cell survival ${ }^{[32]}$, and accelerates oncogene-driven B-lymphoma ${ }^{[33]}$. Suppression of $\mathrm{BCL}-2$, an important mechanism of oncogene-dependent apoptosis, is frequently circumvented during neoplastic B cell transformation in humans and mice ${ }^{[34]}$. Last but not least, our previous work using $B C L 2-$ transgenic mice ${ }^{[35,36]}$ showed that BCL2 selects $\mathrm{B}$ cells harboring deregulated oncogenes for tumor development ${ }^{[4,37]}$.

To compare $\mathrm{BCL}^{+}$dependent lymphoma with a driver of lymphoma development that relies on a fundamentally different mechanism, we chose the $\mathrm{IL}^{+}$transgene. IL- 6 is a pleiotropic pro-inflammatory cytokine that impacts many types of liquid and solid cancers ${ }^{[38]}$, prominently including B-lineage neoplasms such as Hodgkin ${ }^{[39]}$ and non-Hodgkin ${ }^{[40]}$ lymphoma, $\mathrm{PCT}^{[41]}$ and multiple myeloma ${ }^{[42]}$. With regard to tumor development in laboratory mice, IL-6 was first linked to the experimental model system of inflammation-induced peritoneal $\mathrm{PCT}^{[43]}$ - a foundational stone for the design of hybridoma (monoclonal antibody) technology ${ }^{[44]}$. Evidence for the 
pathogenetic significance of IL-6 in PCT development included results in normal $\mathrm{C}$ mice showing that tumor growth was enhanced by exogenous IL-6 but inhibited by antibodies to IL-6 or its receptor ${ }^{[45]}$. In accordance with these findings, IL-6-deficient mice were demonstrated to be resistant to both inflammation-induced $\mathrm{PCT}^{[46]}$ and Myc/Raf retrovirus-induced $\mathrm{PCT}^{[47]}$, whereas IL6 ${ }^{+}$transgenic mice expressing IL-6 widely in B cells and non-B bystander cells in the TME developed PCT spontaneously ${ }^{[28]}$. Furthermore, PCT development in iMyc ${ }^{\mathrm{E \mu}}$ mice, which is driven by deregulated expression of the c-Myc oncoprotein, is greatly accelerated by peritoneal inflammation ${ }^{[48]}$, a process that is known to produce copious amounts of IL-6 at the site of tumor development ${ }^{[49,50]}$. We have recently shown that $\mathrm{iMyc}^{\mathrm{E} \mu}$ - and a similar mouse $M y c$ gene insertion in the Ig heavy-chain locus on Chr 12, called iMyc ${ }^{\mathrm{C} \alpha}$ - collaborates effectively with $\mathrm{IL}^{+}$in malignant plasma-cell transformation ${ }^{[51,52]}$.

When we transferred BCL2 and IL6 onto the genetic background of $A I D$ deficiency and performed tumor induction studies in the newly generated $\mathrm{BCL}^{+} \mathrm{AID}^{-}$and $\mathrm{IL}^{+} \mathrm{AID}^{-}$mice, we fully expected that AID $^{-}$would inhibit lymphoma development in both strains of mice. However, this expectation was only met by $\mathrm{IL}^{+} \mathrm{AID}^{-}$, but not BCL2 ${ }^{+} \mathrm{AID}^{-}$. Our findings in $\mathrm{IL}^{+} \mathrm{AID}^{-}$mice agreed with evidence that AID can initiate lymphomagenesis by virtue of illegitimate genomic rearrangements that occur in B lymphocytes as "accidents" of somatic hypermutation (SHM) or class switch recombination (CSR) independence on AID-mediated cytidine deamination ${ }^{[11]}$. If these accidents do not occur due to loss of AID function, tumor initiation may be held back because it must now rely on an alternative AID-independent mechanism, if it happens at all. An additional mechanism by which AID may have promoted lymphoma development in $\mathrm{IL}^{+}$mice is mutation of non-Ig genes, although this has not been shown here. Thus, AID binds to as many as $\sim 12,000$ sites across the $\mathrm{B}$ cell genome ${ }^{[53]}$, which may cause AID-dependent off-target mutations in non-Ig genes known to be tightly associated with B cell malignancies in humans ${ }^{[54,55]}$. DNA damage repair pathways of AID-dependent U-G mismatches (e.g., base excision repair and DNA mismatch repair) also contribute to malignant $\mathrm{B}$ cell transformation ${ }^{[56]}$. In summary, the weight of evidence on AID-driven lymphoma development in humans and mice supports the interpretation that AID acts as a classic oncogene on the IL $6^{+}$background.

How can we explain the paradoxical observation that AID functions as a tumor suppressor on the BCL2 ${ }^{+}$ background, with loss of AID function ( $\mathrm{AID}^{-}$) promot- ing instead of inhibiting lymphoma? The underlying reason is not known, but it is interesting to note that similar results were obtained using AID $^{-}$mice that carried a $B c l 2 l l^{[9]}, M y c^{[8]}$ or $B C L 6^{[10]}$ transgene. Also, context-dependent dual function of blood cancer genes - either as tumor promoter or tumor suppressor, depending on biological circumstance - has been observed by other investigators in studies on ZEB1 (zinc finger E-box binding homeobox 1$)^{[57]}$, IRF4 (interferon regulatory factor 4$)^{[58]}$ and EZH2 (enhancer of zeste 2 polycomb repressive complex 2 subunit) ${ }^{[59]}$ to name but three examples. Of additional interest is that, a study using $\mathrm{E} \mu c-m y c$ transgenics demonstrated that tumor type and incidence were no different in $\mathrm{AID}^{-}$ $v s$. AID $^{+}$mice $^{[60]}$. AID-transgenic mice spontaneously developed $\mathrm{T}$-cell lymphoma, not B-cell lymphoma as one might have expected from the outset of the study ${ }^{[61]}$. Furthermore, over-expression of AID in the $B$ cell lineage led to mature $B$ cell malignancies only when mice were crossed onto a p53 (TP53)-deficient background $^{[11]}$ - thus abrogating the arguably most important tumor suppressor pathway in mice and humans ${ }^{[62]}$. Additional research is warranted to sort out the mechanism of AID's dual function in neoplastic B cell development, which is not only of interest from a basic research point-of-view but also relevant for new approaches to treat and prevent B cell lymphoma in human beings.

\section{Acknowledgements and funding}

The technical assistance and veterinary care provided by staff of the UI CCOM transgenic mouse facility is gratefully acknowledged. This work was supported by National Natural Science Foundation of China grant 81600177, Natural Science Foundation of Jiangsu Province grant BK20161041 and General Program of University Natural Science Foundation of Jiangsu Province grant 16KJB310009 to CG. This work was also supported by The American Society of Hematology's $7^{\text {th }}$ Round Bridge Grant Program and NIH grants R21CA187388 and R01CA151354 to SJ. Additional support was provided by NCI Core Grant P30CA086862 in support of The University of Iowa Holden Comprehensive Cancer Center.

\section{References}

[1] Kohnken R, Porcu P, Mishra A. Overview of the use of murine models in leukemia and lymphoma research. Front Oncol, 2017,7:22.

[2] Lee EC, Fitzgerald M, Bannerman B, et al. Antitumor activity of the investigational proteasome inhibitor MLN9708 in mouse models of B-cell and plasma cell malignancies. Clin Cancer Res, 2011,17(23):7313-23. 
[3] Tompkins VS, Sompallae R, Rosean TR, et al. Transgenic mouse model of IgM+ lymphoproliferative disease mimicking WaldenstrÖm macroglobulinemia. Blood Cancer J, 2016,6(11):e488.

[4] Strasser A, Harris AW, Cory S. E mu-bcl-2 transgene facilitates spontaneous transformation of early pre$\mathrm{B}$ and immunoglobulin-secreting cells but not $\mathrm{T}$ cells. Oncogene, 1993,8(1):1-9.

[5] Suematsu S, Matsusaka T, Matsuda T, et al. Generation of plasmacytomas with the chromosomal translocation $\mathrm{t}(12 ; 15)$ in interleukin 6 transgenic mice. Proc Natl Acad Sci U S A, 1992,89(1):232-5.

[6] Suematsu S, Matsuda T, Aozasa K, et al. IgG1 plasmacytosis in interleukin 6 transgenic mice. Proc Natl Acad Sci U S A, 1989,86(19):7547-51.

[7] Muramatsu M, Kinoshita K, Fagarasan S, et al. Class switch recombination and hypermutation require activation-induced cytidine deaminase (AID), a potential RNA editing enzyme. Cell, 2000,102(5):553-63.

[8] Kotani A, Kakazu N, Tsuruyama T, et al. Activationinduced cytidine deaminase (AID) promotes B cell lymphomagenesis in Emu-cmyc transgenic mice. Proc Natl Acad Sci U S A, 2007,104(5):1616-20.

[9] Kovalchuk AL, duBois W, Mushinski E, et al. AID-deficient $\mathrm{Bcl}-\mathrm{xL}$ transgenic mice develop delayed atypical plasma cell tumors with unusual Ig/Myc chromosomal rearrangements. J Exp Med, 2007,204(12):2989-3001.

[10] Pasqualucci L, Bhagat G, Jankovic M, et al. AID is required for germinal center-derived lymphomagenesis. Nat Genet, 2008,40(1):108-12.

[11] Robbiani DF, Bunting S, Feldhahn N, et al. AID produces DNA double-strand breaks in non-Ig genes and mature B cell lymphomas with reciprocal chromosome translocations. Mol Cell, 2009,36(4):631-41.

[12] Revy P, Muto T, Levy Y, et al. Activation-induced cytidine deaminase (AID) deficiency causes the autosomal recessive form of the Hyper-IgM syndrome (HIGM2). Cell, 2000,102(5):565-75.

[13] Fagarasan S, Muramatsu M, Suzuki K, et al. Critical roles of activation-induced cytidine deaminase in the homeostasis of gut flora. Science, 2002,298(5597):14247.

[14] Byrd LG, McDonald AH, Gold LG, et al. Specific pathogen-free BALB/c An mice are refractory to plasmacytoma induction by pristane. J Immunol, 1991,147(10):3632-7.

[15] Keim C, Kazadi D, Rothschild G, et al. Regulation of AID, the B-cell genome mutator. Genes Dev, 2013,27(1):1-17.

[16] Morse HC, 3rd, Anver MR, Fredrickson TN, et al. Bethesda proposals for classification of lymphoid neoplasms in mice. Blood, 2002,100(1):246-58.

[17] Feldman AL, Arber DA, Pittaluga S, et al. Clonally related follicular lymphomas and histiocytic/dendritic cell sarcomas: evidence for transdifferentiation of the follicular lymphoma clone. Blood, 2008,111(12):5433-9.

[18] Owen RG, Feyler S, O’Connor SJ, et al. Defining IgM multiple myeloma. Am J Hematol, 2011,86(8):717; author reply 8-9.

[19] Cabrera Q, Chantepie S, Salaun V, et al. IgM multiple myeloma: more on a rare and heterogeneous disease. Am J Hematol, 2011,86(8):717-8; author reply 8-9.

[20] Schuster SR, Rajkumar SV, Dispenzieri A, et al. IgM multiple myeloma: disease definition, prognosis, and differentiation from Waldenstrom's macroglobulinemia. Am J Hematol, 2010,85(11):853-5.

[21] Hirano T, Suematsu S, Matsusaka T, et al. The role of interleukin 6 in plasmacytomagenesis. Ciba Found Symp, 1992,167:188-96; discussion 96-200.

[22] Potter M. Neoplastic development in plasma cells. Immunol Rev, 2003,194:177-95.

[23] Zhang SL, DuBois W, Ramsay ES, et al. Efficiency alleles of the pctr1 modifier locus for plasmacytoma susceptibility. Mol Cell Biol, 2001,21(1):310-8.

[24] Bliskovsky V, Ramsay ES, Scott J, et al. Frap, FKBP12 rapamycin-associated protein, is a candidate gene for the plasmacytoma resistance locus Pctr2 and can act as a tumor suppressor gene. Proc Natl Acad Sci U S A, 2003,100(25):14982-7.

[25] Zhang K, Kagan D, DuBois W, et al. Mndal, a new interferon-inducible family member, is highly polymorphic, suppresses cell growth, and may modify plasmacytoma susceptibility. Blood, 2009,114(14):2952-60.

[26] Potter M, Pumphrey JG, Bailey DW. Genetics of susceptibility to plasmacytoma induction. I. BALB/cAnN (C), C57BL/6N (B6), C57BL/Ka (BK), (C times B6) $\mathrm{F} 1,(\mathrm{C}$ times BK)F1, and $\mathrm{C}$ times $\mathrm{B}$ recombinant-inbred strains. J Natl Cancer Inst, 1975,54(6):1413-7.

[27] Silva S, Kovalchuk AL, Kim JS, et al. BCL2 accelerates inflammation-induced BALB/c plasmacytomas and promotes novel tumors with coexisting $\mathrm{T}(12 ; 15)$ and $\mathrm{T}(6 ; 15)$ translocations. Cancer Res, 2003,63(24):8656-63.

[28] Kovalchuk AL, Kim JS, Park SS, et al. IL-6 transgenic mouse model for extraosseous plasmacytoma. Proc Natl Acad Sci U S A, 2002,99(3):1509-14.

[29] Ranger AM, Malynn BA, Korsmeyer SJ. Mouse models of cell death. Nat Genet, 2001,28(2):113-8.

[30] Bissonnette RP, Echeverri F, Mahboubi A, et al. Apoptotic cell death induced by $\mathrm{c}-\mathrm{myc}$ is inhibited by bcl-2. Nature, 1992,359(6395):552-4.

[31] Evan GI, Wyllie AH, Gilbert CS, et al. Induction of apoptosis in fibroblasts by c-myc protein. Cell, 1992,69(1):119-28.

[32] Vaux DL, Cory S, Adams JM. Bcl-2 gene promotes haemopoietic cell survival and cooperates with c-myc to immortalize pre-B cells. Nature, 1988,335(6189):440-2.

[33] Strasser A, Harris AW, Bath ML, et al. Novel primitive lymphoid tumours induced in transgenic mice by cooperation between myc and bcl-2. Nature, 1990,348(6299):331-3.

[34] Eischen CM, Woo D, Roussel MF, et al. Apoptosis triggered by Myc-induced suppression of Bcl-X(L) or Bcl2 is bypassed during lymphomagenesis. Mol Cell Biol, 2001,21(15):5063-70. 
[35] McDonnell TJ, Deane N, Platt FM, et al. bcl-2-immunoglobulin transgenic mice demonstrate extended $\mathrm{B}$ cell survival and follicular lymphoproliferation. Cell, 1989,57(1):79-88.

[36] Strasser A, Harris AW, Vaux DL, et al. Abnormalities of the immune system induced by dysregulated bcl-2 expression in transgenic mice. Curr Top Microbiol Immunol, 1990,166:175-81.

[37] McDonnell TJ, Korsmeyer SJ. Progression from lymphoid hyperplasia to high-grade malignant lymphoma in mice transgenic for the $\mathrm{t}(14 ; 18)$. Nature, 1991,349(6306):254-6.

[38] Hong DS, Angelo LS, Kurzrock R. Interleukin-6 and its receptor in cancer: implications for translational therapeutics. Cancer, 2007,110(9):1911-28.

[39] Kurzrock R, Redman J, Cabanillas F, et al. Serum interleukin 6 levels are elevated in lymphoma patients and correlate with survival in advanced Hodgkin's disease and with B symptoms. Cancer Res, 1993,53(9):2118-22.

[40] Lam LT, Wright G, Davis RE, et al. Cooperative signaling through the signal transducer and activator of transcription 3 and nuclear factor-\{kappa\}B pathways in subtypes of diffuse large B-cell lymphoma. Blood, 2008,111(7):3701-13.

[41] Nishimoto N, Kanakura Y, Aozasa K, et al. Humanized anti-interleukin-6 receptor antibody treatment of multicentric Castleman disease. Blood, 2005,106(8):2627-32.

[42] Klein B, Tarte K, Jourdan M, et al. Survival and proliferation factors of normal and malignant plasma cells. Int J Hematol, 2003,78(2):106-13.

[43] Potter M, Robertson CL. Development of plasma-cell neoplasms in BALB/c mice after intraperitoneal injection of paraffin-oil adjuvant, heat-killed staphylococcus mixtures. J Natl Cancer Inst, 1960,25:847-61.

[44] Stone MJ. Monoclonal antibodies in the prehybridoma era: a brief historical perspective and personal reminiscence. Clin Lymphoma, 2001,2(3):148-54.

[45] Vink A, Coulie P, Warnier G, et al. Mouse plasmacytoma growth in vivo: enhancement by interleukin 6 (IL6) and inhibition by antibodies directed against IL-6 or its receptor. J Exp Med, 1990,172(3):997-1000.

[46] Lattanzio G, Libert C, Aquilina M, et al. Defective development of pristane-oil-induced plasmacytomas in interleukin-6-deficient BALB/c mice. Am J Pathol, 1997,151(3):689-96.

[47] Hilbert DM, Kopf M, Mock BA, et al. Interleukin 6 is essential for in vivo development of B lineage neoplasms. J Exp Med, 1995,182(1):243-8.

[48] Park SS, Shaffer AL, Kim JS, et al. Insertion of Myc into Igh accelerates peritoneal plasmacytomas in mice. Cancer Res, 2005,65(17):7644-52.
[49] Shacter E, Arzadon GK, Williams J. Elevation of interleukin-6 in response to a chronic inflammatory stimulus in mice: inhibition by indomethacin. Blood, 1992,80(1):194-202.

[50] Hinson RM, Williams JA, Shacter E. Elevated interleukin 6 is induced by prostaglandin E2 in a murine model of inflammation: possible role of cyclooxygenase-2. Proc Natl Acad Sci U S A, 1996,93(10):4885-90.

[51] Rutsch S, Neppalli VT, Shin DM, et al. IL-6 and MYC collaborate in plasma cell tumor formation in mice. Blood, 2010,115(9):1746-54.

[52] Kovalchuk AL, Kim JS, Janz S. E mu/S mu transposition into Myc is sometimes a precursor for $\mathrm{T}(12 ; 15)$ translocation in mouse B cells. Oncogene, 2003,22(18):284250.

[53] Yamane A, Resch W, Kuo N, et al. Deep-sequencing identification of the genomic targets of the cytidine deaminase AID and its cofactor RPA in B lymphocytes. Nat Immunol, 2011,12(1):62-9.

[54] Robbiani DF, Nussenzweig MC. Chromosome translocation, B cell lymphoma, and activation-induced cytidine deaminase. Annu Rev Pathol, 2013,8:79-103.

[55] Casellas R, Basu U, Yewdell WT, et al. Mutations, kataegis and translocations in B cells: understanding AID promiscuous activity. Nat Rev, 2016,16(3):164-76.

[56] Gu X, Booth CJ, Liu Z, et al. AID-associated DNA repair pathways regulate malignant transformation in a murine model of BCL6-driven diffuse large B-cell lymphoma. Blood, 2016,127(1):102-12.

[57] Liu Y, Sanchez-Tillo E, Lu X, et al. The ZEB1 transcription factor acts in a negative feedback loop with miR200 downstream of Ras and Rbl to regulate Bmil expression. J Biol Chem, 2014,289(7):4116-25.

[58] Nam S, Lim JS. Essential role of interferon regulatory factor 4 (IRF4) in immune cell development. Arch Pharm Res, 2016,39(11):1548-55.

[59] Sashida G, Iwama A. Multifaceted role of the polycomb-group gene EZH2 in hematological malignancies. Int J Hematol, 2017,105(1):23-30.

[60] Nepal RM, Zaheen A, Basit W, et al. AID and RAG1 do not contribute to lymphomagenesis in Emu c-myc transgenic mice. Oncogene, 2008,27(34):4752-6.

[61] Okazaki IM, Hiai H, Kakazu N, et al. Constitutive expression of AID leads to tumorigenesis. J Exp Med, 2003,197(9):1173-81.

[62] Schmitt CA, McCurrach ME, de Stanchina E, et al. INK4a/ARF mutations accelerate lymphomagenesis and promote chemoresistance by disabling p53. Genes Dev, 1999,13(20):2670-7.

(Received 17 September 2017, Revised 22 October 2017, Accepted 28 October 2017) 